\section{Incidence and severity of iris pigmentation on latanoprost-treated glaucoma eyes}

S-Y Chou, C-K Chou, T-M Kuang and W-M Hsu
Department of

Ophthalmology

Taipei Veterans General

Hospital National

Yang-Ming University

Taipei, Taiwan

Correspondence: C-K Chou

Department of

Ophthalmology

Taipei Veterans General

Hospital National Yang-

Ming University

Taipei

11217, Taiwan

Tel: + 886228757325

Fax: + 886228761351

E-mail: chou1231@

ms28.hinet.net

Received: 22 August 2003 Accepted: 1 June 2004

Published online:

3 September 2004

\begin{abstract}
Purpose The purpose of the present study was to investigate the incidence and severity of iridial pigmentation under latanoprost topical use on brown eyes in Taiwan.

Methods Retrospective review study was conducted from April 1999 to October 2001 in the Department of Ophthalmology, Taipei

Veterans General Hospital, Taiwan, for glaucoma clinic monthly follow-up patients; 140 open-angle glaucoma patients on $\mathbf{0 . 0 0 5 \%}$ latanoprost were enrolled. Analyses of iridial pigmentation incidence, grading, patient age distribution, side effect, and time course were performed. Boys-Smith pigment gradation lens was used as standard for semiquantitative iris pigmentation grading.

Result Before $\mathbf{0 . 0 0 5 \%}$ latanoprost use, $\mathbf{9 0 \%}$ of the patients enrolled were noted with iridial pigmentation grade $I$, and $10 \%$ were with grade I-II, but not reaching grade II scale standard. A total of 60 patients on $0.005 \%$ latanoprost developed increased pigmentation of the iris during the follow-up period. An increase of iris pigmentation was noted after an average of 7.27 months use of latanoprost (range 1-19 months, SD 2.65 months). For iridial pigmentation grading, 57.1, 30.7, 10.0, and $2.1 \%$ of our patients were noted to have grade I, II, III, and IV respectively. Most patients with latanoprost-induced iris hyperpigmentation were with grade II iridial pigmentation. There were 15 patients (10.7\%) (10 female and five male) with hypertrichosis in the study group who were not compatible with the iridial pigmentation status. Among these patients, female patients had higher incidence of hypertrichosis than males, but this did not bother them. Only four patients (2.8\%) were with conjunctiva chemosis and three patients $(2.1 \%)$ with lid margin hyperpigmentation.
\end{abstract}

Conclusion Contrary to the belief that latanoprost rarely caused iris hyperpigmentation in yellow-brown eyes, our study showed that $42.8 \%$ iris hyperpigmentation did occur, especially after continual use for around 7 months. Higher hyperpigmentation incidence were noted in male than in female patients. This might be due to stronger adrenergic incidence in male than in female patients. Although hypertrichosis and increasing eyelid pigmentation together with iridial pigmentation represented a potentially permanent cosmetic side effect, they are very rare and occurred in no more than $3 \%$ in our patients. It is a good way to take Boys-Smith pigment gradation lens for iridial pigmentation grading and for long-term continual evaluation. The doctors should exert great care in differentiating drug-induced iris pigmentation and iris nevi from early stage uveal melanoma.

Eye (2005) 19, 784-787. doi:10.1038/sj.eye.6701663; published online 3 September 2004

Keywords: latanoprost; iridial

hyperpigmentation; Boys-Smith gradation lens

Introduction

Latanoprost, a prostaglandin F2 alpha analogue, is a recent antiglaucomatous drug that has been reported to induce a discrete incidence of increased iridial pigmentation as a side effect in some patients. ${ }^{1}$ Theoretically, this would be due to increased melanogenesis or melanocyte proliferation. $^{2}$ Similar effects were seen in 12, 23, and $11 \%$ of patients in the USA, United Kingdom (UK), and Scandinavia, respectively, during 1 year of treatment. The highest incidence of induced pigmentation was 
seen in green-brown, yellow-brown, and

blue/grey-brown eyes, in that order. ${ }^{3}$ However, no iridial pigmentation-grading study had been reported in the Medline for long-term evaluation. We proposed the use of Boys-Smith pigment gradation lens as standard for iridial pigmentation grading and long-term follow-up and comparison.

\section{Patients and methods}

This is a retrospective study in which 280 eyes of 140 primary open-angle glaucoma patients were collected. All patients were under $0.005 \%$ latanoprost treatment and were regularly followed up in the Glaucoma Clinic of Taipei Veterans General Hospital from April 1999 to October 2001. The patients were all under regular monthly follow-up in this period.

We used the Boys-Smith pigment gradation lens (Boys-Smith lens, Ocular Instruments, Bellevue, WA, USA) to measure semiquantitatively the amount of pigment visible in the iris of patients under $0.005 \%$ latanoprost. To determine semiquantitatively the amount of pigment in the iris, we compared the color of the iris with the five-pigment column on the Boys-Smith lens. ${ }^{4}$ In the gonioscopic lens, column pigments representing the gradation were incorporated as a five-density pigment $(0$, I, II, III, IV) scales so that the human iris and the scale could be simultaneously viewed through the gonioscopy mirror using the same illumination (Figure 1a). A routine ophthalmic examination, including measurement of intraocular pressure with the Goldmann applanation tonometer, was performed at every visit. Our patients were all examined under the same criteria of Boys-Smith pigment gradation lens pigmented grading by the same senior glaucoma specialist. Patients using more than one kind of topical antiglaucoma drugs were excluded. We also excluded patient who was previously diagnosed with uveitis, heterochromic iridocyclitis, and diabetes mellitus. Iridial pigmentation incidence, grading, time course, and other side effects were evaluated.

Iridial pigmentation grading were recorded with Boys-Smith lens grading before and after latanoprost use. The area of hyperpigmentation was divided into three grades: mild scattered-hyperpigmentation involving $\leqslant 1 / 3$ of the area from limbus to pupil margin; moderately scattered-hyperpigmentation involving $>1 / 3$ but $\leqslant 2 / 3$ of the area from limbus to pupil margin, and diffuse type.

\section{Results}

There were 85 male and 55 female patients. The average age of male patients was 65.4 years (range 12-84) and that of female patients was 65.0 years (range 15-82).
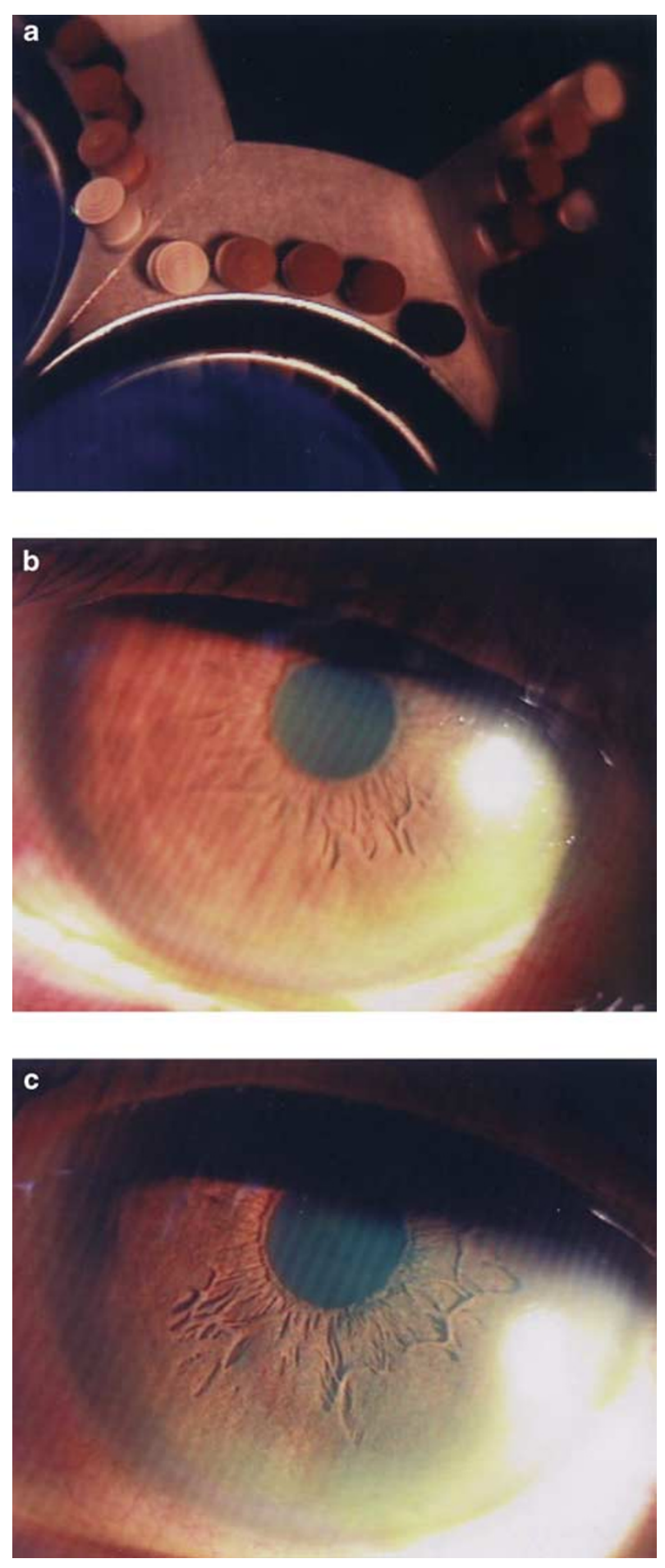

Figure 1 (a) Boys-Smith gonioscopic trabecular meshwork pigmentation grading (0-IV). (b) Grade I iris hyperpigmentation before latanoprost used. (c) Iris hyperpigmentation involving about half of the area from limbus to pupil margin (moderately scattered). Hyperpigmentation typically started from the 6 o'clock position and progressed concentrically to the 12 o'clock position. 
Before latanoprost use, $90 \%$ of the patients enrolled were with iridial pigmentation grade I, the remaining $10 \%$ of them were with grade I-II but not reaching grade II scale standard. In total, 60 patients on latanoprost $0.005 \%$ developed increased pigmentation of the iris, with an incidence of $42.8 \%$ after 1-year of follow-up. An increase of iris pigmentation appeared after an average of 7.27 months use of latanoprost (range 1-19 months, SD 2.65 months). Nobody with brown-yellow eyes was grade 0 in our patients. For iridial pigmentation grading 0, 57.1, $30.7,10.0$, and $2.1 \%$ of patients were with grade 0 , I, II, III, and IV, respectively (Table 1). Most patients with latanoprost-induced iris hyperpigmentation were grade II (30.7\%). It was noted that more male patients had iris hyperpigmentation than female patients $(1.55: 1)$ $(P<0.05)$. Two of our patients on $0.005 \%$ latanoprost revealed grade III iris pigmentation after 1-month use and one patient with grade IV iris hyperpigmentation after 2-month use; these three patients were all males.

Almost all patients were unaware of their iridial hyperpigmentation. Iris hyperpigmentation in our patient group was grossly inconspicuous: only by careful slit-lamp examination can one identified the hyperpigmented condition. Hyperpigmentation usually started from the inferior part of the iris near the limbus (6 o'clock position) and was observed to progress upward towards the pupil margin as well as in a concentrically upward direction towards the 12 o'clock position. In all, $20.6 \%$ of the patients were in the mild scattered group, $66.7 \%$ in the moderately scattered group, and $12.7 \%$ in the diffuse hyperpigmented group. Hypertrichosis with higher eyelashes growth density was noted in 15 patients $(10.7 \%)$ on $0.005 \%$ latanoprost after 1 year. This was not compatible with their iridial pigmentation status. Among those who developed hypertrichosis, there were more female patients (10 patients) than males (five patients). On the other hand, four patients (2.8\%) had conjunctival

Table 1 Relationship between iris pigmentation grade, period, and incidence

\begin{tabular}{llcc}
\hline $\begin{array}{l}\text { Iris } \\
\text { pigmentation } \\
\text { grade }\end{array}$ & Gender $(\mathrm{n}=140)$ & $\begin{array}{c}\text { Age } \\
\text { (mean) } \\
\text { (years) }\end{array}$ & $\begin{array}{c}\text { Time since } \\
\text { latanoprost } \\
\text { used (months) } \\
\text { Mean (SD) }\end{array}$ \\
\hline 0 & 0 & & \\
I & Male 47 (33.5\%) & 65.31 & $7.28(2.55)$ \\
II & Female 33(23.6\%) & 64.65 & \\
& Male 24 (17.2\%) & 65.04 & $7.27(2.65)$ \\
III & Female 19(13.5\%) & 65.96 & \\
& Male 11(7.85\%) & 64.89 & $8.07(4.19)$ \\
IV & Female 3(2.14\%) & 63.76 & $12.67(4.61)$ \\
& Male 3(2.14\%) & 65.82 & \\
& Female None & None & \\
& Total 140 & & $7.01(3.67)$ \\
\hline
\end{tabular}

Table 2 Incidence of side effects besides iridial hyperpigmentation after latanoprost used

\begin{tabular}{lccc}
\hline Side effect & $\begin{array}{c}\text { Male } \\
(\mathrm{n}=85)\end{array}$ & $\begin{array}{c}\text { Female } \\
(\mathrm{n}=55)\end{array}$ & $\begin{array}{c}\text { Total numbers } \\
(\mathrm{n}=140)\end{array}$ \\
\hline Hypertrichosis & 10 & 5 & 15 \\
Eyelid hyperpigmentation & 2 & 1 & 3 \\
Conjunctiva hyperaemia & 2 & 2 & 4 \\
Itchy & 0 & 2 & 2 \\
\hline
\end{tabular}

chemosis and three patients (2.1\%) had lid margin hyperpigmentation (Table 2). Although the combination of hypertrichosis, increased eyelid pigmentation with iridial hyperpigmentation represents a potentially permanent cosmetic side effect, the incidence were very low (less than $3.0 \%$ ) in our study group.

\section{Discussion}

From the literature, xalatan-induced iris hyperpigmentation were noted in 12,23 , and $11 \%$ of patients in the USA, UK, and Scandinavia, respectively, during 1 year of treatment. The highest incidence of induced pigmentation was seen in green-brown, yellow-brown, and blue/grey-brown eyes, in that order. It was also noted that irides, homogenously blue, grey, green, or brown, were seldomly affected. ${ }^{3}$

In the study of brown-yellow eyes in Japan, the incidence of iris pigmentation was $51.6 \%$ at 12 months after use and our study group showed $42.8 \% .^{5,6}$ Two of our patients on $0.005 \%$ latanoprost revealed grade III iris pigmentation after 1-month use and one patient with grade IV iris hyperpigmentation after 2-month use; they were all males.

Contrary to the belief that latanoprost rarely causes iris hyperpigmentation in dark brown eyes, our study showed that latanoprost does cause iris hyperpigmentation with an incidence of $42.8 \%$ in 1-year follow-up.

Iris colour can be affected by a variety of ocular disorders. It is suspected that iris colour may not remain constant throughout life. Differences in the iris colour of normal eyes are the result of variable amounts of melanin pigment granules within a constant number of melanocytes in the superficial stroma of iris. Diseases such as Horner syndrome and Fuchs' heterochromic iridocyclitis affect iris colour, resulting in a decrease of iris pigmentation. Application of the prostaglandin analogue latanoprost, on the other hand, leads to an increase in iris pigmentation in some patients. ${ }^{7}$ Latanoprost is an ester product analogue of prostaglandin F2 alpha, which effectively reduces intraocular pressure by increasing uveoscleral outflow 
rather than altering conventional trabeculo-canalicular aqueous outflow. The intraocular pressure lowering effect of latanoprost lasts for 20-24 h after a single dose. Latanoprost is well tolerated with no, or barely detectable, conjunctiva hyperaemia and unlike timolol, is not associated with systemic adverse effect. ${ }^{8}$ It is noted that $3-10 \%$ of patients treated with latanoprost showed increased iris pigmentation after 3-4.5 months. Other local side effects include local irritation, increased growth of eyelashes (hypertrichosis) and a possible association with cystoid macula oedema ${ }^{3,9}$

Adrenergic influence on iris pigmentation in newborn rabbits has been studied before and adrenergic influence on iris stroma melanogenesis appear to be mediated by alpha-adrenergic receptors. ${ }^{10}$ There are more veterans in our patients and hence more male than female patients. But it was still noted that males have higher incidence with heavy pigmentation than female patients. The period with iridial hyperpigmentation formation is around 7 months on average (range 1-19 months) and the other study with brown-yellow eyes revealed typically, a concentric increase of the iris pigmentation after 6 months (range $3-17){ }^{3}$ Both are comparable. It is a good approach to take Boys-Smith pigment gradation lens for iris hyperpigmentation grading in further evaluation and follow-up. After cessation of latanoprost, no change of the induced pigmentation has been found for 1.5 years. It was suggested that latanoprost-induced stimulation of melanin production follows the metabolic pathway involving tyrosinase activity and may be relevant for the therapeutic application of latanoprost in glaucoma in order to reduce its side effect resulting in increased iris pigmentation. ${ }^{1}$

We had 15 patients $(10.7 \%)$ with hypertrichosis noted in our study group (10 female and five male patients) and related iridial pigmentation grading; three female cases with grade I showed hypertrichosis. Female patients had higher incidence of hypertrichosis than males but this did not bother the patients at all. Most patients with latanoprost-induced pigmentation are grade II $(30.7 \%)$ and grade III (10.0\%). Only four patients (2.8\%) with conjunctiva chemosis and three patients $(2.1 \%)$ with lid margin hyperpigmentation, and they are all female patients. Although hypertrichosis, increased eyelid pigmentation $^{5}$ together with iridial pigmentation represents a potentially permanent cosmetic side effect associated with the use of this highly effective intraocular pressure-lowering agent, ${ }^{3}$ this is rare. Owing to lacking systemic effect noted in our study group and in previous reports, latanoprost remains an important therapeutic drug in the management of patients with primary openangle glaucoma. The doctors should be careful in differentiating drug-induced iris pigmentation and iris nevi from early stage uveal melanoma.

\section{References}

1 Drago F, Marino A, La Manna C. alpha-Methyl-p-tyrosine inhibits latanoprost-induced melanogenesis in vitro. Exp Eye Res 1999; 68: 85-90.

2 Dutkiewicz R, Alber DM, Levin LA. Effects of latanoprost on tyrosinase activity and mitotic index of cultured melanoma lines. Exp Eye Res 2000; 70: 563-569.

3 Wistrand PJ, Stjernschantz J, Olsson K. The incidence and time-course of latanoprost-induced iridial pigmentation as a function of eye color. Surv Ophthalmol 1997; 41: 129-138.

4 Sugiyama K, Kitazawa Y. Trabecular pigmentation following extracapsular cataract extraction and posterior chamber intraocular lens implantation. Ophthalmic Surg 1990; 21: 700-703.

5 Kook MS, Lee K. Increased eyelid pigmentation associated with use of latanoprost. Am J Ophthalmol 2000; 129: 804-806.

6 Hara T. Nippon Ganka Gakkai Zasshi: Increased iris pigmentation after use of latanoprost in Japanese brown eyes. Acta Soc Ophthalmol Jpn 2001; 105: 314-321.

7 Imesch PD, Wallow IH, Albert DM. The color of the human eye: a review of morphologic correlates and of some conditions that affect iridial pigmentation. Surv Ophthalmol 1997; 41: 117-123.

8 Patel SS, Spencer CM. Latanoprost: a review of its pharmacological properties, clinical efficacy and tolerability in the management of primary open-angle glaucoma and ocular hypertension. Drugs Aging 1996; 9: 363-378.

9 Hejkal TW, Camras CB. Prostaglandin analogs in the treatment of glaucoma. Semin Ophthalmol 1999; 14: 114-123.

10 Odin L, O'Donnel Jr FE. Adrenergic influence on iris stromal pigmentation: evidence for alpha-adrenergic receptors. Invest Ophthalmol Vis Sci 1982; 23: 528-530. 